\title{
Query complexity for searching multiple marked states from an unsorted database
}

\author{
Bin Shand* \\ School of Computer Science \& Technology,Beijing Institute of Technology, \\ No. 5, Zhongguancun Nandajie, Haidian District, Beijing 100081, P. R. China
}

(Dated: October 8, 2018)

\begin{abstract}
An important and usual problem is to search all states we want from a database with a large number of states. In such, recall is vital. Grover's original quantum search algorithm has been generalized to the case of multiple solutions, but no one has calculated the query complexity in this case. We will use a generalized algorithm with higher precision to solve such a search problem that we should find all marked states and show that the practical query complexity increases with the number of marked states. In the end we will introduce an algorithm for the problem on a "duality computer" and show its advantage over other algorithms.
\end{abstract}

\section{INTRODUCTION}

Since L. K. Grover 1] 2] discovered the quantum algorithm for the unsorted database search problem with single marked state, many improvements have been made on it. Among those, Michel Boyer et al 3 ] were the first to generalize it to the case of multiple solutions; G. Brassard et al [4], P. Høyer [5], G. L. Long [6] respectively improved Grover's algorithm and obtained certainty in finding the single marked state in different ways. Besides, many works [3 [7] 8] 9] [0] analyzed the query complexity and lower bounds of Grover's algorithm or related algorithms for search problems. However, the query complexity for searching all multiple marked states from an unsorted database has not been addressed. This problem is very important when recall is emphasized in searching multiple objects.

\section{QUERY COMPLEXITY FOR SEARCHING ALL MULTIPLE MARKED STATES USING GENERALIZED LONG'S ALGORITHM}

\author{
Generalized Long's algorithm
}

A generalization of Long's algorithm $[6]$ for searching single marked state with certainty in an unsorted database to the case of multiple marked states can be easily shown like this: repeat Long's algorithm until all marked states have been found.

\section{Problem description}

Let us define the problem as: Under randomized conditions, search all $m$ marked states $\left|\tau_{1}\right\rangle,\left|\tau_{2}\right\rangle, \ldots,\left|\tau_{m}\right\rangle$ from an unsorted database with $N$ states $|0\rangle,|1\rangle, \ldots,\left|\tau_{1}\right\rangle,\left|\tau_{2}\right\rangle, \ldots,\left|\tau_{m}\right\rangle, \ldots,|N-1\rangle$ with no less than overall probability of success $1-\delta$.

\section{Solution and query complexity}

Now we analyze the number of queries needed to solve this problem. Without loss of generality, we divide the process to find all $m$ marked states into $m$ steps. We devote $q_{i}$ as the queries needed and $r_{i}$ as the times that we should run Long's algorithm, to find $\left|\tau_{i}\right\rangle$; we devote $q$ as the total queries needed, and $r$ as the total times that we should run Long's algorithm. Besides, for simplicity we suppose we could "fortunately" find every marked state ultimately during corresponding queries in every step. Still, we can educe the query complexity with high precision if $\delta$ is small.

\section{Step 1}

Without loss of generality, we can find $\left|\tau_{1}\right\rangle$ within $q_{1}=$ $\mathcal{O}\left(\sqrt{\frac{N}{m}}\right)$ queries with certainty using Long's algorithm. We should run the algorithm for only once, so here $r_{1}=$ 1.

Step 2

We should stress that in this step we have probability $\frac{1}{m}$ to find $\left|\tau_{1}\right\rangle$ again in the first run of Long's algorithm; if it occurs, we have to start the second run of the algorithm, and thus we have probability $\left(\frac{1}{m}\right)^{2}$ to find $\left|\tau_{1}\right\rangle$ again.... As a result, to find $\left|\tau_{2}\right\rangle$ with probability $1-\delta$,we should run Long's algorithm for $r_{2}$ times such that

$$
\frac{m-1}{m}+\frac{1}{m} \times \frac{m-1}{m}+\left(\frac{1}{m}\right)^{2} \times \frac{m-1}{m}+
$$

$$
\ldots+\left(\frac{1}{m}\right)^{r_{2}} \times \frac{m-1}{m}=1-\delta
$$

which equals to

$$
\left(\frac{1}{m}\right)^{r_{2}}=\delta
$$


Thus, we can get

$$
r_{2}=\frac{\ln \delta^{-1}}{\ln m}
$$

and

$$
\begin{gathered}
q_{2}=\frac{\ln \delta^{-1}}{\ln m} \mathcal{O}\left(\sqrt{\frac{N}{m}}\right) \\
\ldots \ldots
\end{gathered}
$$

Step $i$

In this step we have probability $\frac{i-1}{m}$ to find what we have found in former steps- $\left|\tau_{1}\right\rangle,\left|\tau_{2}\right\rangle, \ldots,\left|\tau_{i}-1\right\rangle$-again in the first run of Long's algorithm; if it occurs, we have to start the second run of the algorithm, and thus we have probability $\left(\frac{i-1}{m}\right)^{2}$ to find what we have found in former steps again.... As a result, to find $\left|\tau_{i}\right\rangle$ with probability $1-\delta$,we should run Long's algorithm for $r_{i}$ times such that

$$
\begin{gathered}
\frac{m-i+1}{m}+\frac{1}{m} \times \frac{m-i+1}{m}+\left(\frac{1}{m}\right)^{2} \times \frac{m-i+1}{m}+ \\
\ldots+\left(\frac{1}{m}\right)^{r_{i}} \times \frac{m-i+1}{m}=1-\delta
\end{gathered}
$$

which equals to

$$
\left(\frac{i-1}{m}\right)^{r_{i}}=\delta
$$

Thus, we can get

$$
r_{i}=\frac{\ln \delta^{-1}}{\ln m-\ln (i-1)}
$$

and

$$
\begin{gathered}
q_{i}=\frac{\ln \delta^{-1}}{\ln m-\ln (i-1)} \mathcal{O}\left(\sqrt{\frac{N}{m}}\right) \\
\ldots \ldots
\end{gathered}
$$

Step $m$

In this step we have probability $\frac{m-1}{m}$ to find what we have found in former steps again in the first run of Long's algorithm; if it occurs, we have to start the second run of the algorithm, and thus we have probability $\left(\frac{m-1}{m}\right)^{2}$ to find what we have found in former steps again.... As a result, to find $\left|\tau_{m}\right\rangle$ with probability $1-\delta$, we should run Long's algorithm for $r_{m}$ times such that

$$
\frac{1}{m}+\frac{m-1}{m} \times \frac{1}{m}+\left(\frac{m-1}{m}\right)^{2} \times \frac{1}{m}+
$$

$$
\ldots+\left(\frac{m-1}{m}\right)^{r_{m}} \times \frac{1}{m}=1-\delta
$$

which equals to

$$
\left(\frac{m-1}{m}\right)^{r_{m}}=\delta
$$

Thus, we can get

$$
r_{m}=\frac{\ln \delta^{-1}}{\ln m-\ln (m-1)}
$$

and

$$
q_{m}=\frac{\ln \delta^{-1}}{\ln m-\ln (m-1)} \mathcal{O}\left(\sqrt{\frac{N}{m}}\right)
$$

\section{Result}

To add up, we can obtain

$$
\begin{array}{r}
r=1+\sum_{k=1}^{m-1} \ln \delta^{-1} \ln ^{-1}\left(\frac{m}{k}\right) \\
q=\left[1+\sum_{k=1}^{m-1} \ln \delta^{-1} \ln ^{-1}\left(\frac{m}{k}\right)\right] \mathcal{O}\left(\sqrt{\frac{N}{m}}\right)
\end{array}
$$

\section{Examples}

Let us see two examples of the result of $r$.

\section{Example 1}

We set $\delta=0.01$, and devote

$$
f(m)=1+\sum_{k=1}^{m-1} \ln 0.01^{-1} \ln ^{-1}\left(\frac{m}{k}\right)
$$

$f(m)$ is plotted in FIG 1 and FIG 2

\section{Example 2}

We set $m=1000$, and devote

$$
f(\delta)=1+\sum_{k=1}^{999} \ln \delta^{-1} \ln ^{-1}\left(\frac{1000}{k}\right)
$$

$f(\delta)$ is plotted in FIG 3 and FIG 4

From these figures, we can conclude that the query complexity will increase rapidly with increase of the number of the marked states at a specific probability of success, and will increase with precision we need i.e. probability of success in finding every marked state. 


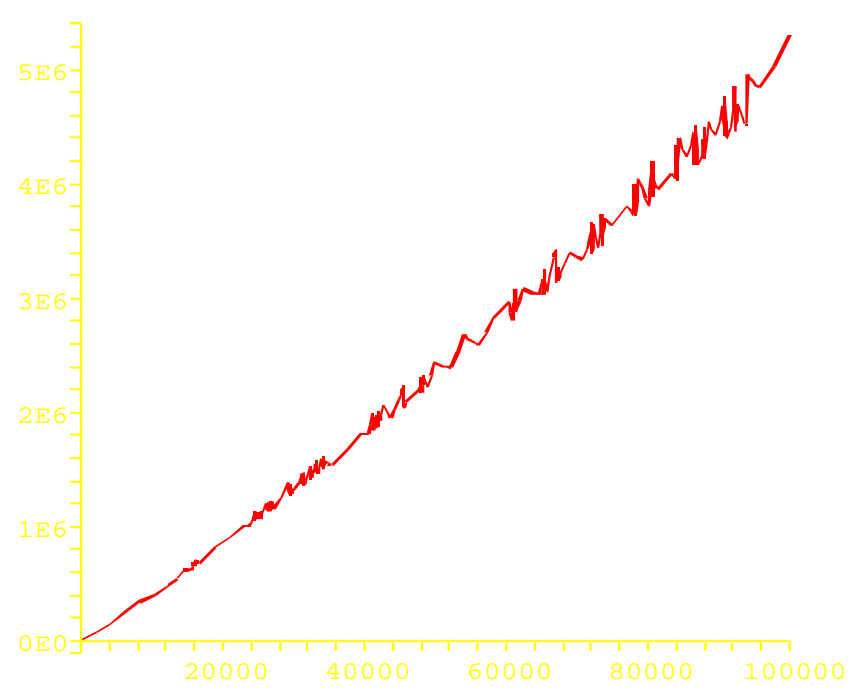

FIG. 1: $f(m)$ when $\delta=0.01,1 \leq m \leq 100000$

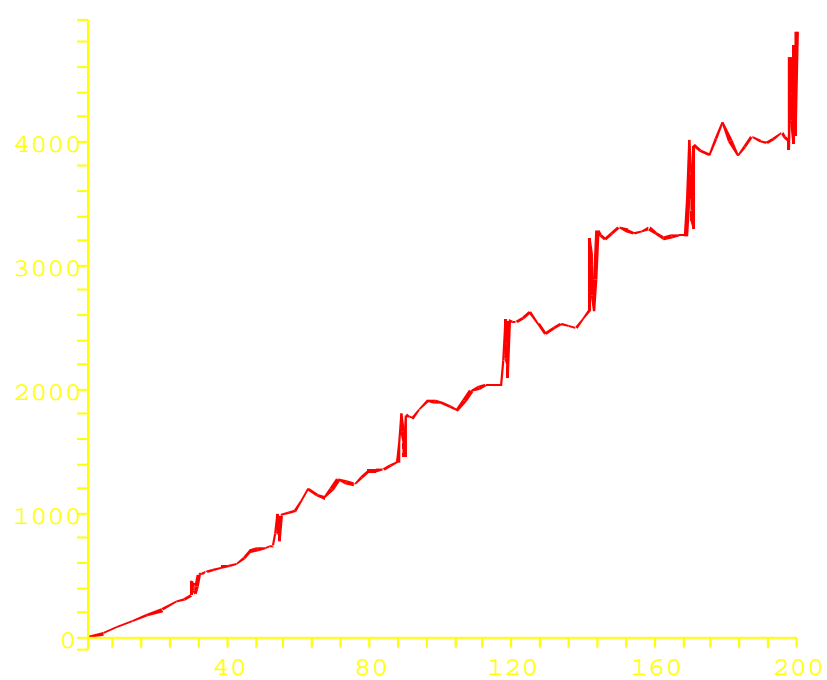

FIG. 2: $f(m)$ when $\delta=0.01,1 \leq m \leq 200$

\section{THE SEARCH PROBLEM ON A DUALITY COMPUTER}

G. L. Long proposed a new quantum computing model-duality computer 11$]$-utilizing quantum system's wave-particle duality which can achieve exponential speedup on unsorted database search problems. Long proposed as well two search algorithms on a duality computer respectively for the case of single marked state 12



FIG. 3: $f(\delta)$ when $m=1000,0.00001 \leq \delta \leq 0.5$

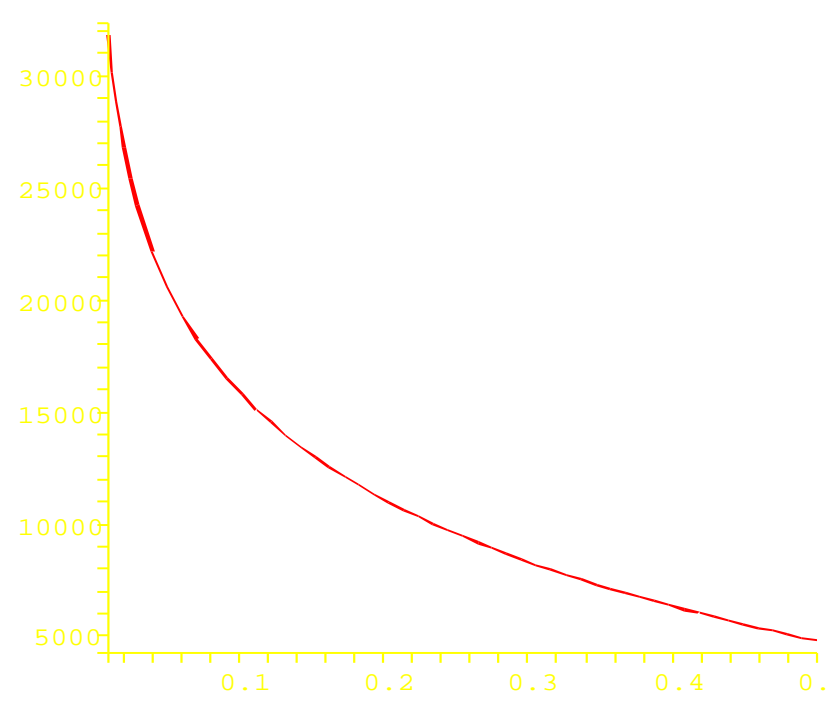

FIG. 4: $f(\delta)$ when $m=1000,0.01 \leq \delta \leq 0.5$

and the case of multiple marked states. In the latter case, one can find all $m$ marked states from an unsorted database with $N$ states with certainty within $m \log \frac{N}{m}$ queries using $\log N$ "dubits".

We should stress that in a duality computer, we can easily — within time and space complexity $\mathcal{O}(1)$ - delete the marked states that we have found from the initial states. So we will not meet such problems that with $m$ increasing the query complexity increases as well when 
searching multiple marked states.

\section{ACKNOWLEDGEMENTS}

The author would like to thank Gui Lu Long for very helpful discussion and thank Jia $\mathrm{Qu} \mathrm{Yi}$ for his generous help in plotting the function figures.

* binshang@bit.edu.cn

[1] L. K. Grover, A fast quantum mechanical algorithm for database search, Proceedings of 28th Annual ACM Symposium on Theory of Computing, 1996, pp. 212-219.

[2] L. K. Grover, Quantum mechanics helps in searching for a needle in a haystack, Physical Review Letters, Vol. 79, no. 2, 14 July 1997, pp. 325-328.

[3] Michel Boyer, Gilles Brassard, Peter Høyer and Alain Tapp,Tight bounds on quantum searching", Fortschritte Der Physik, specialissue on quantum computing and quantum cryptography, 1998, Vol. 46,pp. 493-505.
[4] G. Brassard, P. Ho.yer, M. Mosca, and A. Tapp, quant-ph/0005055

[5] P. Høyer, Phys. Rev. A 62, 052304, 2001.

[6] G. L. Long, Grover algorithm with zero theoretical failure rate,Phys. Rev. A, Vol. 64, 022307, 2001. quant$\mathrm{ph} / 010607$.

[7] Beals, Robert, Harry Buhrman, Richard Cleve, Michele Mosca and Ronald de Wolf, Quantum lower bounds by polynomials, Proceedings of 39th Annual Symposium on Foundations of Computer Science,November 1998, pp. $352 \mathrm{C} 361$

[8] A. Ambainis. Quantum lower bounds by quantum arguments, J. Comput. Sys. Sci. 64:750-767, 2002. quant-ph/0002066

[9] P. Høyer, J. Neerbek, and Y. Shi. Quantum complexities of ordered searching, sorting, and element distinctness. Algorithmica, 34(4):429-448, 2002.

[10] C.Zalka, Grovers quantum searching algorithm is optimal, Phys. Rev. A60, 2746 1999, quant-ph/9711070

[11] G. L. Long. The general quantum interference principle and the duality computer(2005), to appear in Commun.Theor.Phys. quant-ph/0512120

[12] B. Shang. A summary on two new algorithms for Grover's unsorted database search problem. quant-ph/0603210 